\title{
Recombinant Human Hyaluronidase
}

National Cancer Institute

\section{Source}

National Cancer Institute. Recombinant Human Hyaluronidase. NCI Thesaurus. Code C61504.

A human recombinant form of the naturally occurring human enzyme hyaluronidase with potential chemoadjuvant activity. Upon local administration, recombinant human hyaluronidase hydrolyzes hyaluronic acid, a glucosaminoglycan responsible for the viscosity of the interstitial barrier. The digestion of hyaluronic acid lowers the viscosity in the interstitial space, thereby increasing permeability and facilitating local penetration of chemotherapeutic agents into cancer cells. 